\title{
Smart Hospital-Room and Modern Photonics Emerging Clinical Reality Based on Optical Systems
}

\author{
Basile Spyropoulos \\ Department of Biomedical Engineering, Technological Education Institute (TEI) of Athens, Athens, Greece
}

Email address:

basile@teiath.gr

\section{To cite this article:}

Basile Spyropoulos. Smart Hospital-Room and Modern Photonics Emerging Clinical Reality Based on Optical Systems. Optics. Vol. 7, No. 1, 2018, pp. 18-31. doi: 10.11648/j.optics.20180701.14

Received: December 9, 2017; Accepted: December 27, 2017; Published: January 19, 2018

\begin{abstract}
Numerous solutions have been proposed for the emerging "Smart Hospital-Room", however, there is little progress made yet. Interesting approaches include the Amplion Smart (er) Room of the Future, the IBM-UPMC (University of Pittsburgh Medical-Center), the NXT-Health-program of the US-Department of Defense and the EU-Agency for Network and Information Security. Nevertheless, a Hospital Smart-Room has to be cost-effective and it is the aim of this project, to pinpoint the most important catches. Crucial delay-factors and high-cost sources have been traced, by following the innovation trail, as reflected upon numerous Hospital-Technology related Patents and other research Publications, synopsizing the obstacles for achieving an acceptable and interoperable Electronic Medical Record and the enhanced Cognitive-computing based ways to harness, share, manage and trace big-data. On the other hand, increased Patient-interactivity and participation, is a cardinal challenge for the future's Smart-Room and the Health-care providers need access to real-time, Point-of-care feedback, on a continuous basis. Finally, Technology supporting overloaded clinical-staff causes frequently complications and tension. Therefore, Clinical-workflow improvements require goal-oriented training, based on Patient-care-plans and supported by mature and cost-effective Technologies. Cost, lack of education and short-term focusing lead to cul-de-sac. The Hospitalleaders must realize that the world is changing dramatically; in order to survive, they have to offer Smart, innovative, efficient and above all affordable and cost-effective Health-care. Modern Photonics may improve dramatically the smooth operation of the "Smart Hospital-Room", as it is proven in the detailed created "Patent mappings" relating Photonics Technology and Health-care.
\end{abstract}

Keywords: Medical Records, Cognitive-Computing, Patient-Participation, Goal-Oriented Training, Patient Care-Plan, Patents, Patent-Mappings

\section{Introduction}

Numerous solutions have been proposed for the future "Smart Hospital-Room", however, little progress has been made. Interesting approaches include the Amplion Smart (er) Room of the Future, the IBM-UPMC (University of Pittsburgh Medical-Center), the NXT-Health-program of the US-Department of Defense and the EU-Agency for Network and Information Security [1].

A Hospital Smart-Room has to be cost-effective and it is the aim of this project, to pinpoint the most important financial and health-care related "catches".

Crucial delay-factors and high-cost sources have been traced, by following the innovation trail, as reflected upon numerous Hospital-Technology related Publications and
Patents, synopsizing:

1. The obstacles for achieving an acceptable and interoperable Electronic Medical Record.

2. Enhanced cognitive-computing based ways to harness, share, manage and trace big-data.

3. Increased Patient-interactivity and participation, is a cardinal challenge for future's Smart-Room.

4. Health-care providers need access to real-time, point-ofcare feedback, on a continuous basis.

5. Technology supporting overloaded clinical-staff causes sometimes complications and tension.

6. Clinical-workflow improvements require goal-oriented training, on Patient-care-plans, supported by mature and cost-effective technologies. 


\section{Crucial Delay-Factors and High-Cost Sources}

A. The obstacles for achieving an acceptable and interoperable Electronic Medical Record (EMR)

EMR implementation is being delayed for decades and was a "black-hole" for money, out of the Hospital and the "Smartroom". Installation, management and training of EMR have decelerated the advances elsewhere, within Hospitals. [2], [3].

The EMR providers are finally beyond that and hopefully they will start acting also on other technology initiatives. NXT Patient Room 2020 won a national design-award; however, this is beyond the reality, for almost all Hospitals.

Looking at the existing or near-term technologies and other attainable innovations in Interoperability, Big-data, Patients' engagement and Clinical-workflow, will help us get Smarter.

As experience with EMR implementations increases, new knowledge is gained, on how to make these implementations more successful.

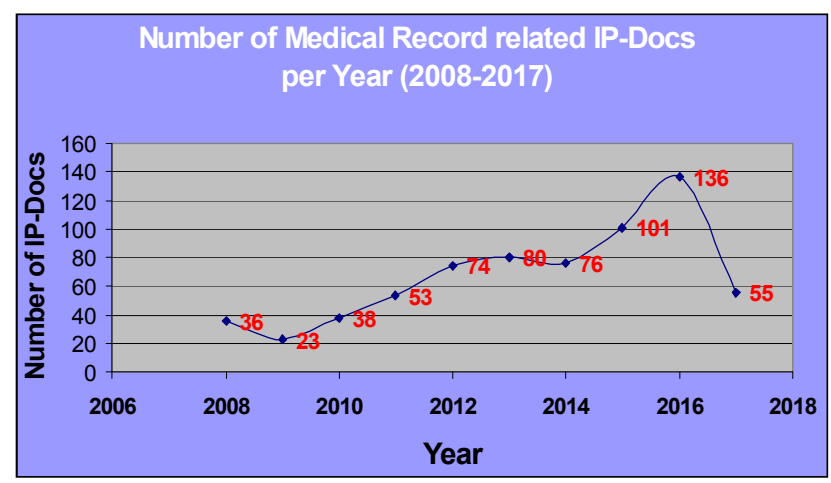

Figure 1. Number of EMR related IP-Docs per Year.
New conceptual frameworks, described in the relevant Medical-Technical literature, provide a richer understanding of what makes an EMR implementation successful.

B. Enhanced cognitive-computing based ways to harness, share, manage and trace Big-data

In 2013, Joel Dudley, Director of Biomedical Informatics for the Mount Sinai Medical School, shared a vision for Big- data and its potential, to dramatically impact the healthcare system. As he compared Face-book's capabilities in harnessing analytics and insights, within the "social network", compared to the "clinical network", he was frustrated by Healthcare industry's progress [1].

Despite the explosive data-employment in Healthcare, we have not leveraged it, for key Patient-insights and real-time work-flows. We are still not enough competent to make datawork for Medicine and modern Healthcare.

Reducing costs through the use of Big-data include highcost Patients, Re-admissions, Triage, Decompensation, Adverse-events Registry and Treatment optimization, for Diseases and Conditions, affecting multiple Organ-systems.

The "Smart-Room" needs to provide Proactive, and eventually, Predictive care, as described detailed, in Figure 2. Predictive Analytics are common applications, including also Health-care potential applications.

C. Reducing costs through the use of Big-data

There is plenty of equipment in a Hospital Smart-room, creating Data, as shown in Figure 3. However, only 41 Big-data relevant Patent-applications have been filed during the period 208-2017, according to our search (cf. Figure 1).

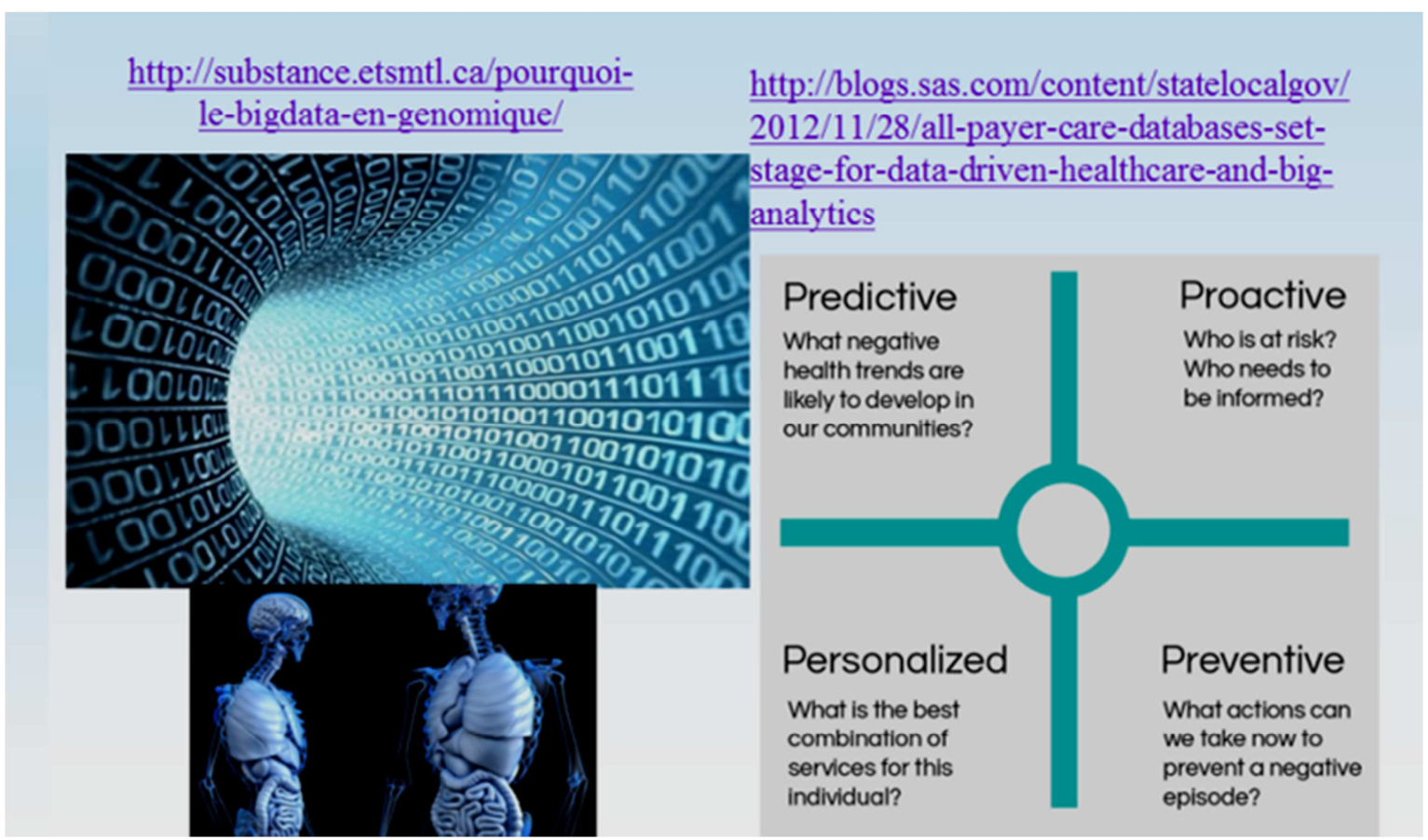

Figure 2. Reducing costs through the use of big data [4], [5]. 


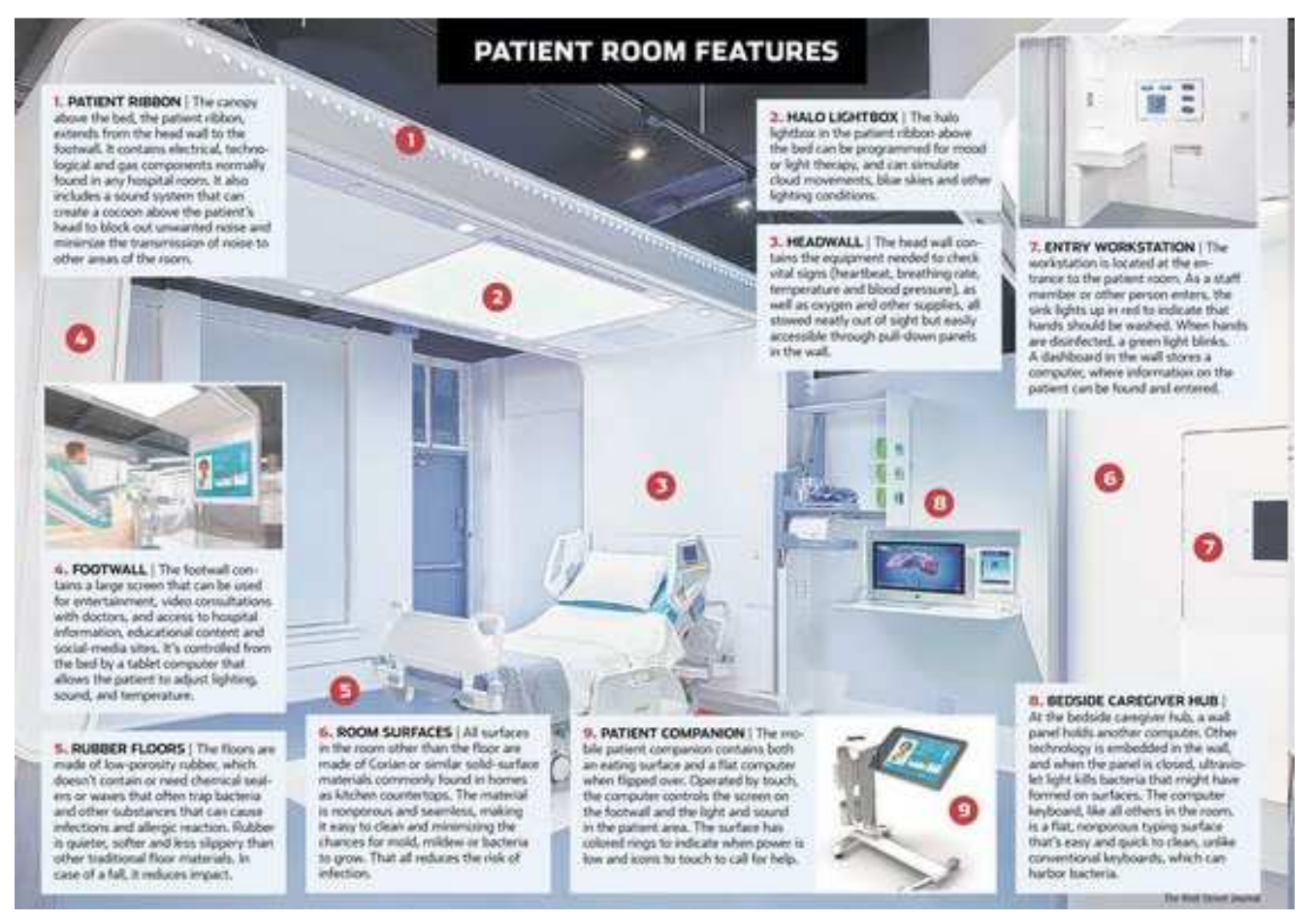

Figure 3. Reducing costs through the use of big data [6], [7], [8].

D. Increased Patient-interactivity and participation is a cardinal challenge for future's Smart-Room

With interoperable technology and more effective data practices still pending, Hospitals must follow more closely the Patients, to improve their experience and their engagement. A Smart-room enhances the ability of Patients to be actively involved. The emerging Smart-room, will allow the Patients and their families, to see what's going on, with their Care.

Enabling Patients' engagement, through a Smartphone-app is a genius, low-cost and high-impact way toward their dynamic Communication and Education. We need to move faster, concerning Ubiquitous Mobile-technology, when it comes up to Patients' Engagement and Instruction. Hospitals should learn from the Hotel-industry about customerservice...

Hospitals must advance beyond traditional paper-based "customer satisfaction surveys", where feedback is limited and delayed up to six months after it has been collected.

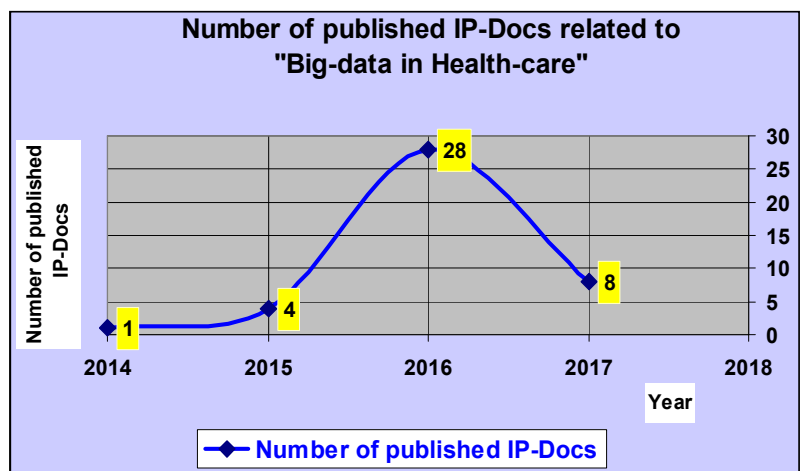

Figure 4. Number of published IP-Docs related to "Big-data in Healthcare".

\section{E. Supporting Patient's interactivity \& engagement}

Health-care providers need access to real-time Point-ofcare feedback, on a continuous basis. The Point of Care Programme aims to transform the quality of Patients' experience in acute Hospitals. Achieving this ambitious goal will depend on the active engagement of all Medical Nursing and other Personnel that has direct contact with Patients [9], [10].

They in turn must require stronger support from their Managers, Trustees and Commissioners. All these interested parties, need robust evidence, on the quality of Patients' experience, and that of their families.

It is now widely recognised that the best source of this information is the Patients and their families.

There is a big empty space omitted here, why?

F. Improving Patient experience in primary care

A multi-method program of research on the measurement and improvement of Patient experience is available in https:/www.ncbi.nlm.nih.gov/pubmed/28654227 [11].

There has been an increased focus towards improving quality of care within the UK-NHS and other Health-care systems, in the last 15 years. The development and administration of large-scale National Patient-surveys to gather representative data, on Patient experience, such as the National GP-Patient Survey, in primary care, has been such an initiative.

However, it remains unclear how the survey is used by Patients and what impact the data may have on Practices, and more specific:

1. Understanding Patient-experience data.

2. Acknowledging know-how in minority Ethnic-groups.

3. Using Patients' go-through data.

Findings demonstrate the importance of Patients' 
experience feed-back, as a means of informing Health-care Systems and confirm that Surveys are a rather useful resource for monitoring trends in Quality of Care.

G. Improving Patient experience in primary care

Technology supporting overloaded clinical-staff causes sometimes complications and tension. Technology improving workflow can't increase workload.
As we embrace technology towards integration, leveraging big-data, connecting more deeply with Patients, we can't forget the Clinical-team, responsible for delivering the Care.

Is it possible at all, to create a more efficient and effective workflow, for Clinical-staff that fosters support, accountability and improved satisfaction, both for caregivers and Patients?

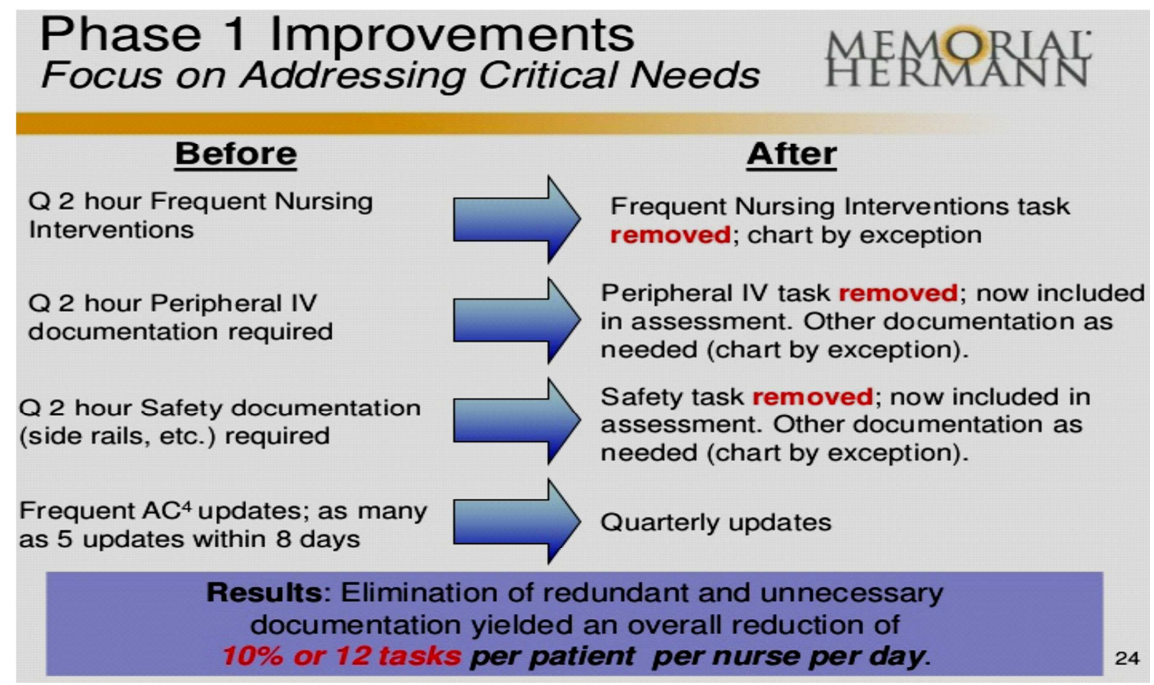

Figure 5. Reducing the personnel work-load by introducing targeted improvements [12].

Nurses are over-worked, challenged to do more than is by any means possible, in one shift. Frequently, Technology designed to make life easier, for Clinical-staff, may instead create more complications and anxiety.

This is one of the main reasons, why so much Technology goes unused, underused or even misused, and why Hospital leaders are skeptical about adopting additional and expensive Biomedical Technology Innovations.

\section{Clinical-Workflow Improvements}

In the absence of useful technology, Nurses may become very creative and they have a long history of being very inventive, in absence of needed Medical Equipment and Tools.

There is a big empty space omitted here, why?

To move toward new "Smart Room Concepts" for Clinical- workflow, Hospitals must focus on assisting and supporting Nurses, with initiating, updating, following and meeting goals, for Patients' Care-plans, by leveraging existing and cost-effective technologies. There are tools that improve the management of Workflow and the tracking of Activity. Some of these tools, have been around for a while, but they have not been always seriously adopted and implemented [13], [14].

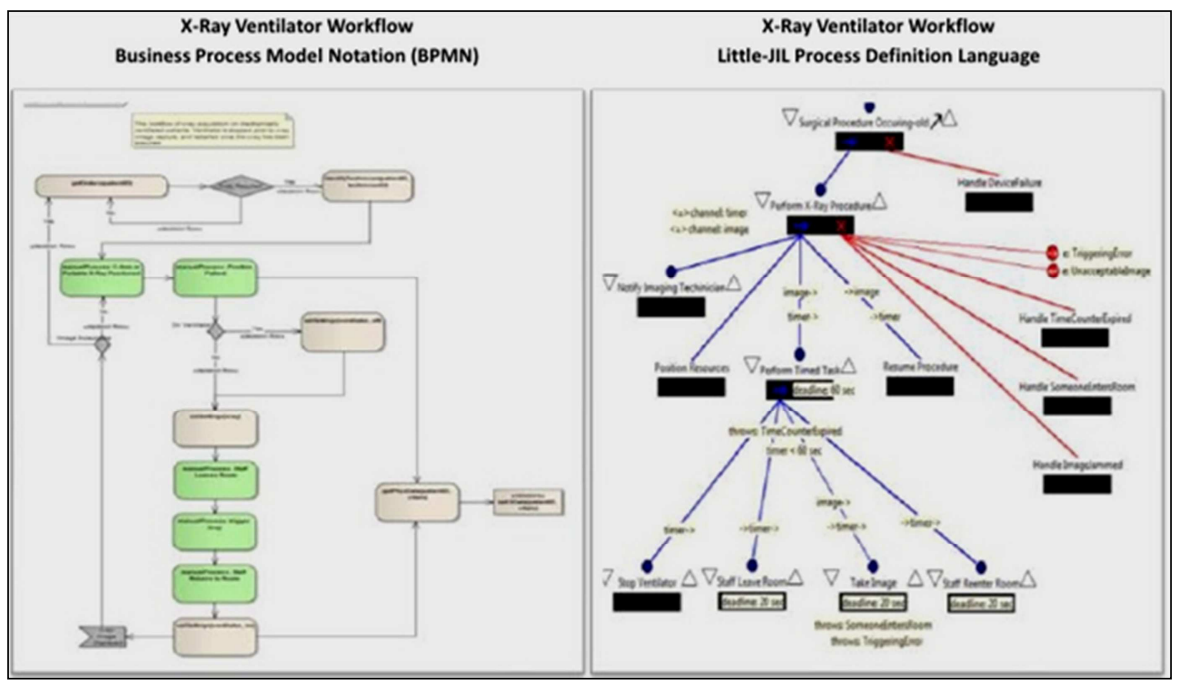

Figure 6. The two views of a Clinical Process [14]. 


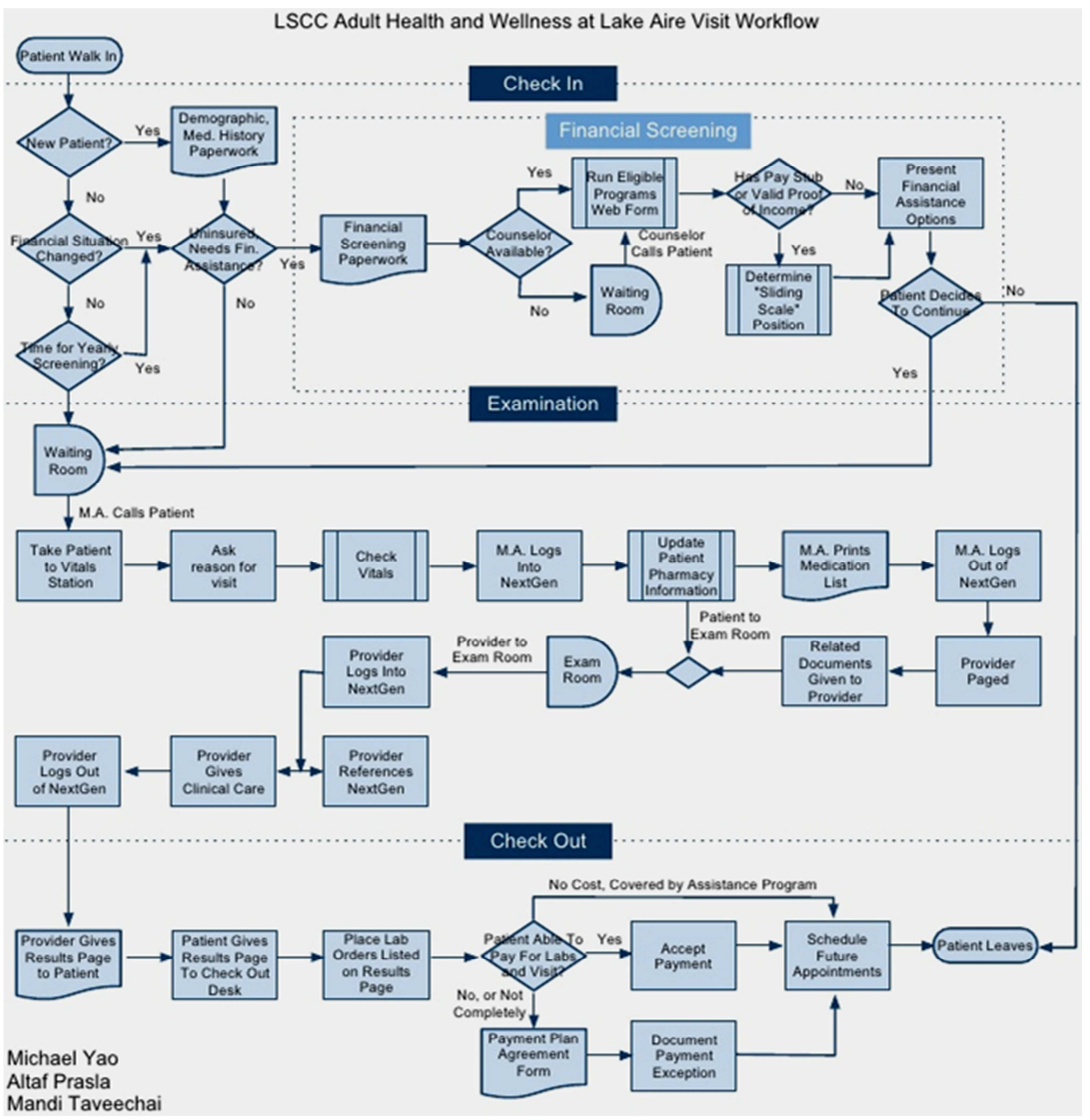

Figure 7. A typical Clinical-workflow diagram [13].

What's really holding us back from Smarter Patient rooms? The answer seems to be: Goal-oriented training on Patient-care plans, supported by mature and cost-effective technologies.

\section{Some of the Smart Room Obstacles}

Budget constraints, especially outside of Academic Institutions, exclude the way of consistent Innovation, because of shortages in cash-flow. Short term focus-in, instead of investing in long-term strategies, involving the people with the most influence on every day Patients' Care, the Nurses.
Hospitals have to think about the "Smart-room" of the "future", rather as a step-by-step process. This is the reason that Interoperability is so critical, for Equipment and Software.

We have to continuously build toward the "Smart Room" to be able to add on "modules" and expand over time. Most Hospitals do not have budget and staff to achieve now right away implementation, however, many could be ready to take the first steps.

The "Smart Room" is not an object that one organization should, or even could own; it is rather a "array" of various Technologies, Solutions and Companies that can cooperate and integrate, in favor of Patients' Care. 


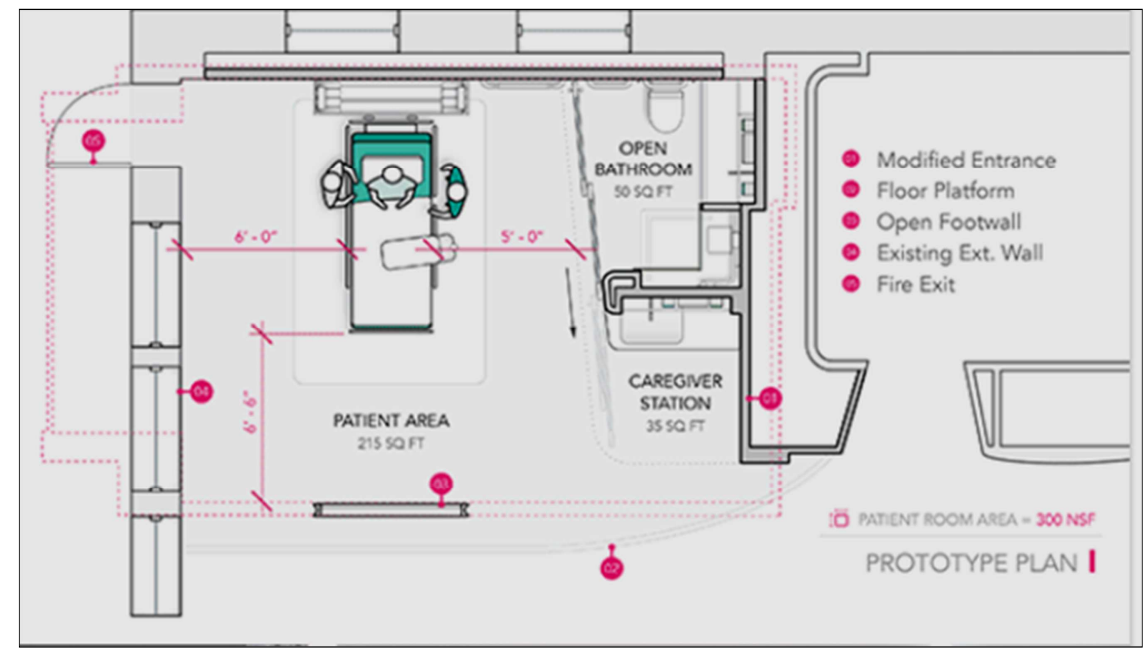

Figure 8. A typical Hospital Smart-room lay-out [15].

\section{Photonics Employment in the Smart Hospital-Room}

Modern Photonics may improve dramatically the smooth operation of the "Smart Hospital-Room", as it is proven in the detailed created "Patent mappings" relating Photonics Technology and Health-care. A patent map is a graphical model of patent visualization. This practice enables companies to identify the patents in a particular technology space, verify the characteristics of these patents, and identify the relationships among them, to see if there are any zones of infringement. Global Patent Map reveals the Structure of Technological Progress.

By mapping the way patents cite each other, network scientists have been able to study how different technologies rely on each other and how new technologies emerge. The created Figures. 1, 4, 9-14 and 17 display typical Opticsrelated patent-mappings of Industrial Property Documents (IP-Docs). The term Photonics, has been developed, as an outgrowth of the first practical Semiconductor light emitters, that were invented in the early ` 60 s and the Optical fibers in the 70 s.

Though covering all light's technical applications over the whole spectrum, most photonic applications are in the range of visible and near-infrared light. Light sources used in Photonics are usually far more sophisticated than light bulbs. Photonics commonly use semiconductor light sources like light-emitting diodes (LEDs), super luminescent diodes, and LASERs.

Other light sources include single photon sources, fluorescent lamps, cathode ray tubes (CRTs), and plasma screens. While CRTs, plasma screens, and organic lightemitting diode displays, generate their own light, liquid crystal displays (LCDs) like TFT screens require a backlight of either, cold cathode fluorescent lamps or, more often today, LEDs.

Characteristic for research on semiconductor light sources is the frequent use of III-V semiconductors instead of the classical semiconductors like Silicon and Germanium (compare Figures 9-14).

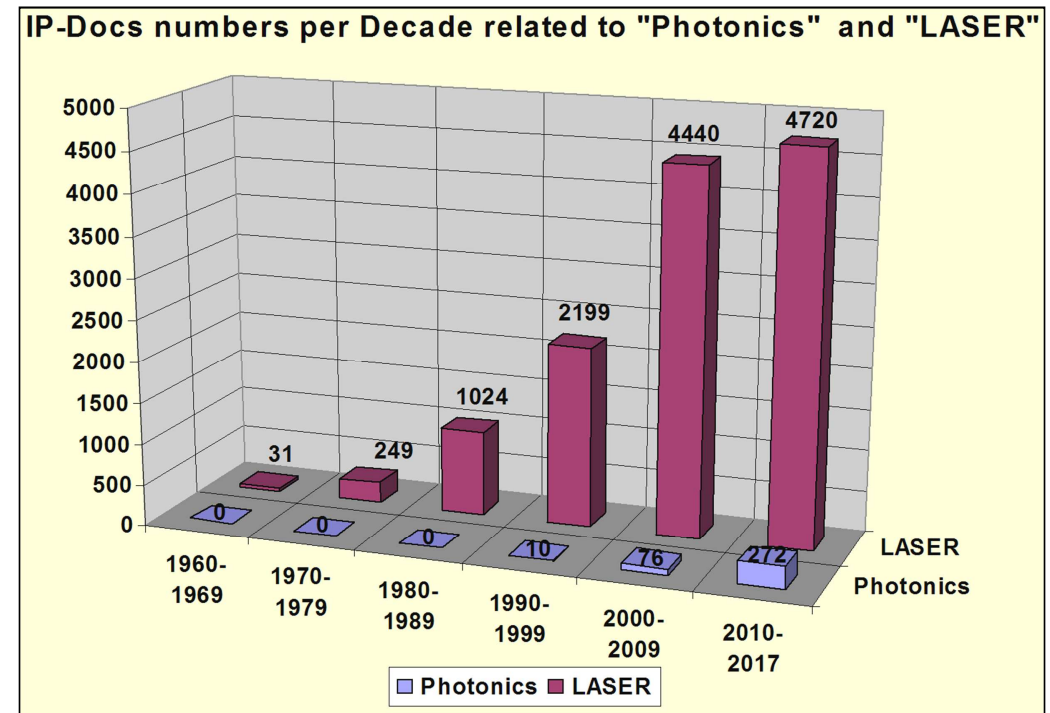

Figure 9. Results of an extended Patent-search, concerning Patent-applications related to LASERs and Photonics (1960-2017) performed by employing the European Patent Organization (EPO) search-engine esp@cenet. 


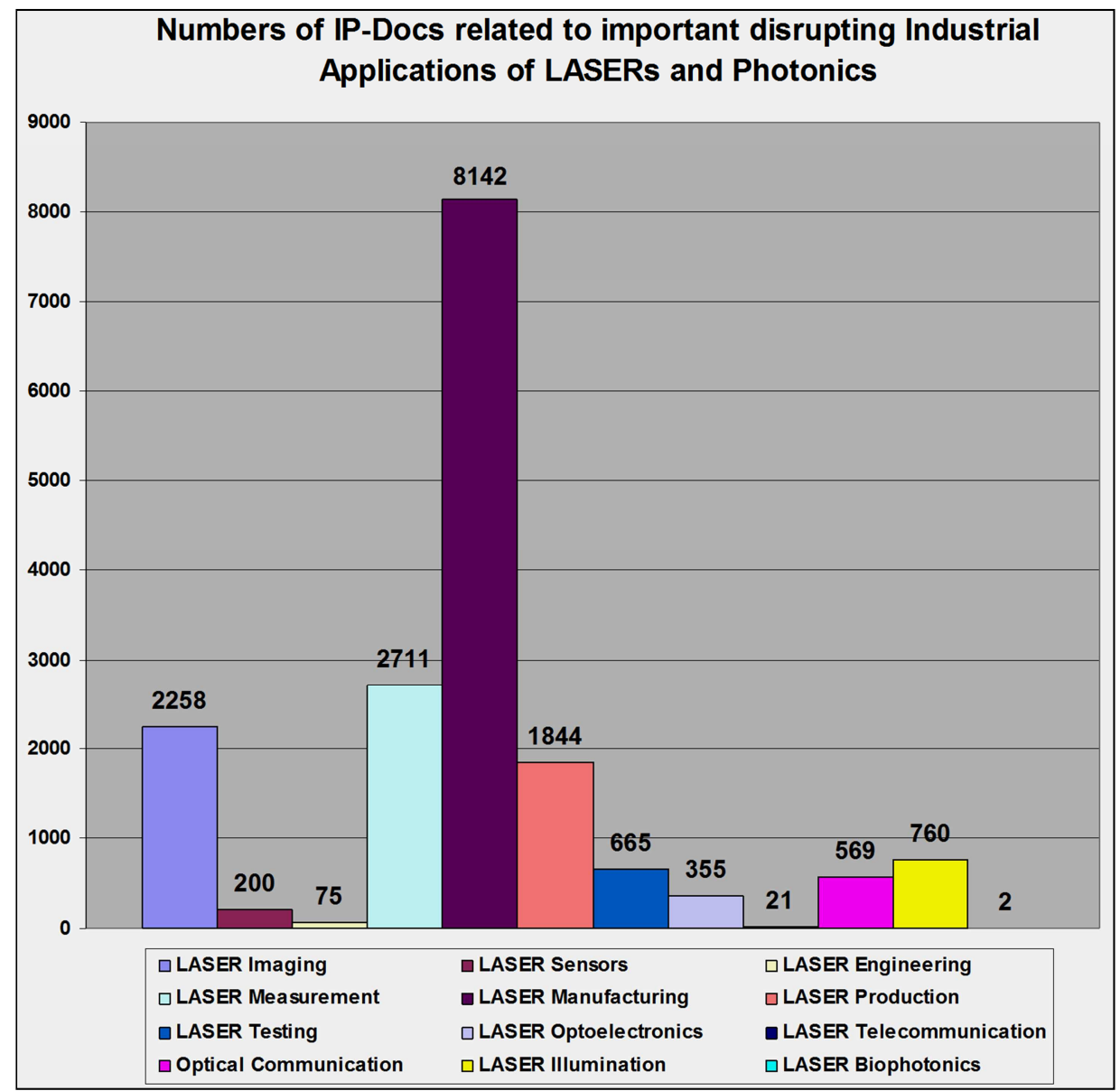

Figure 10. Results of an extended Patent-search, concerning Patent-applications related to the most important aspects of LASERs and Photonics Industrial applications.

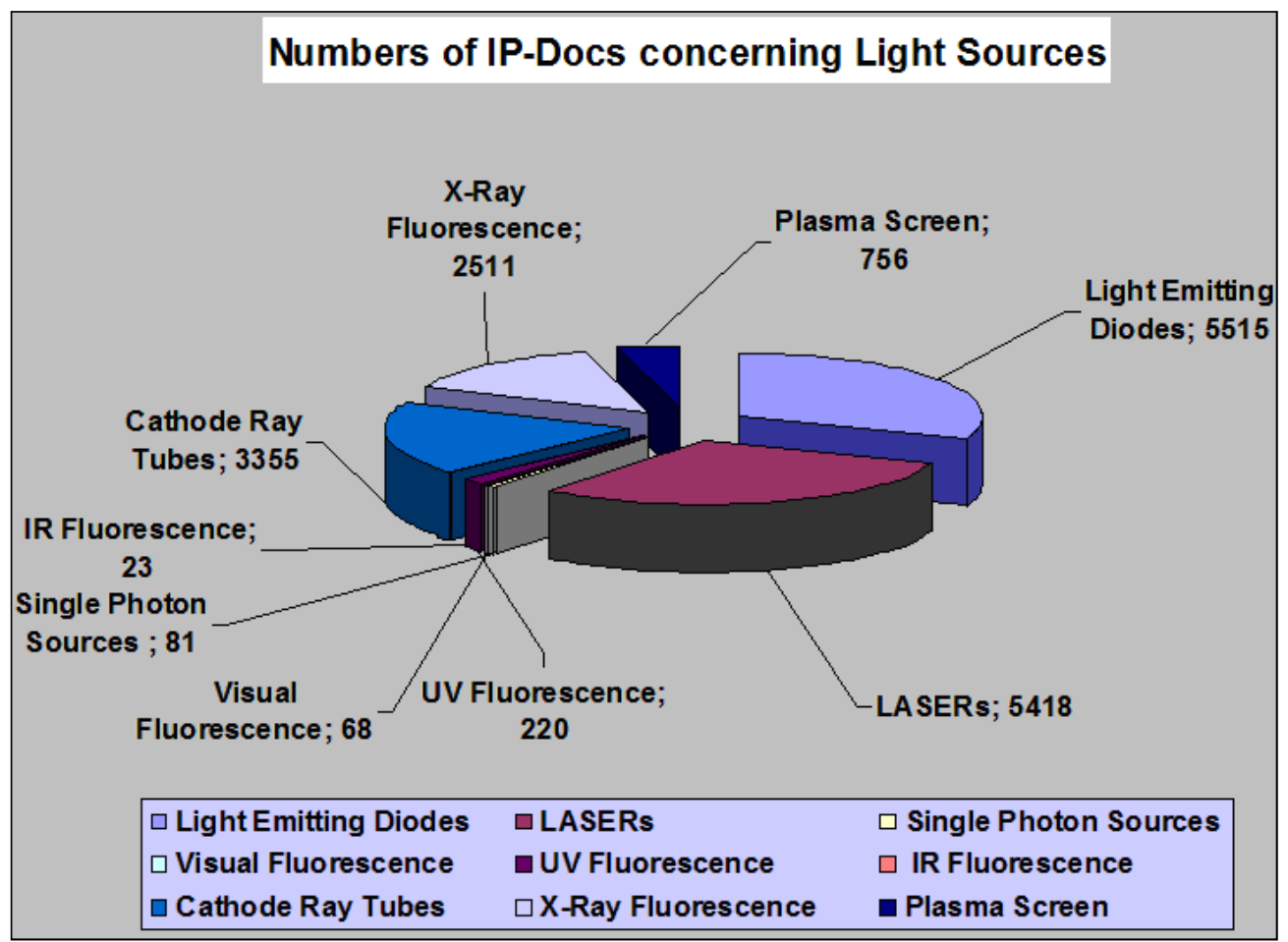

Figure 11. Numbers of IP-Docs concerning the most important Light Sources. 


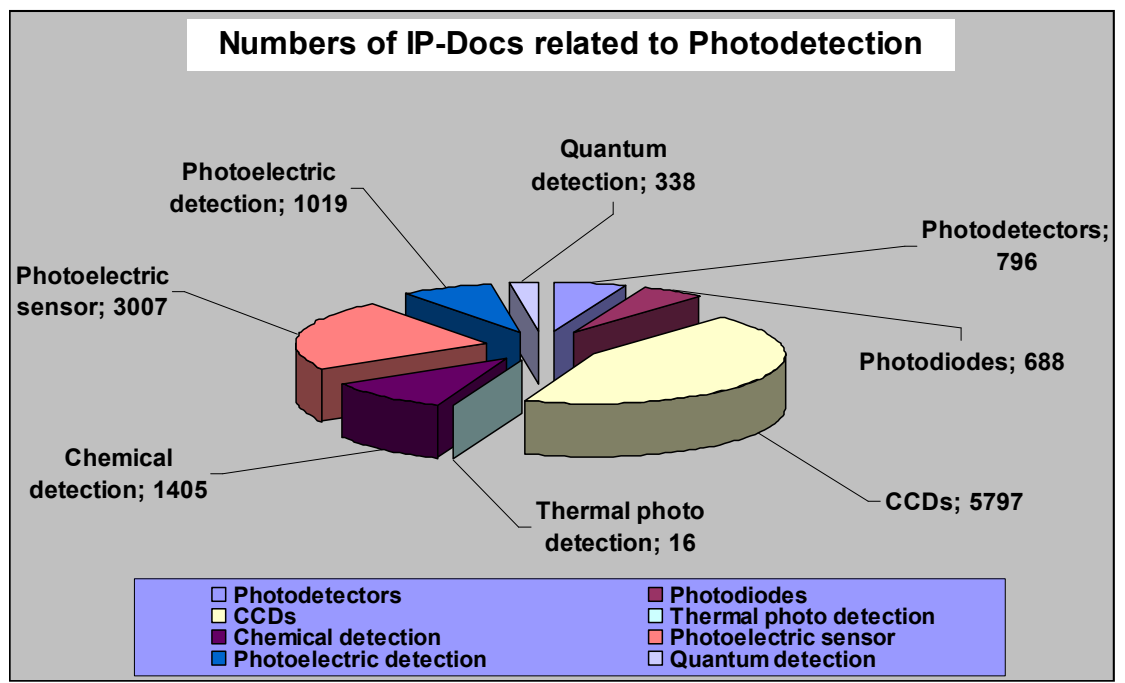

Figure 12. Numbers of IP-Docs concerning the most important Light Sources.

This is due to the special properties of III-V semiconductors that allow for the implementation of light emitting devices. Examples for material systems used are Gallium Arsenide (GaAs) and Aluminum Gallium Arsenide (AlGaAs) or other compound semiconductors. They are also used in conjunction with Silicon, to produce hybrid SiLASERs.

Light can be transmitted through any transparent medium. Glass fiber or plastic optical fiber can be used to guide the light along a desired path. In optical communications optical fibers allow for transmission distances of more than $100 \mathrm{~km}$ without amplification depending on the bit rate and modulation format used for transmission (Figure 13).

A very advanced research topic within Photonics is the investigation and fabrication of special structures and "materials" with engineered optical properties. These include photonic crystals, photonic crystal fibers and meta-materials. Optical amplifiers are used to amplify an optical signal. Optical amplifiers used in optical communications are
Erbium-doped fiber amplifiers, semiconductor optical amplifiers, Raman amplifiers and optical parametric amplifiers (Figure 14). A very advanced research topic on optical amplifiers is the research on quantum dot semiconductor optical amplifiers.

Photodetectors detect light. Photodetectors range from very fast photodiodes for communications applications over medium speed charge coupled devices (CCDs) for digital cameras to very slow solar cells that are used for energy harvesting from sunlight. There are also many other photodetectors based on thermal, chemical, quantum, photoelectric and other effects. Modulation of a light source is used to encode information on a light source.

Modulation can be achieved by the light source directly. One of the simplest examples is to use a flashlight to send Morse code. Another method is to take the light from a light source and modulate it in an external optical modulator. An additional topic covered by modulation research is the modulation format.

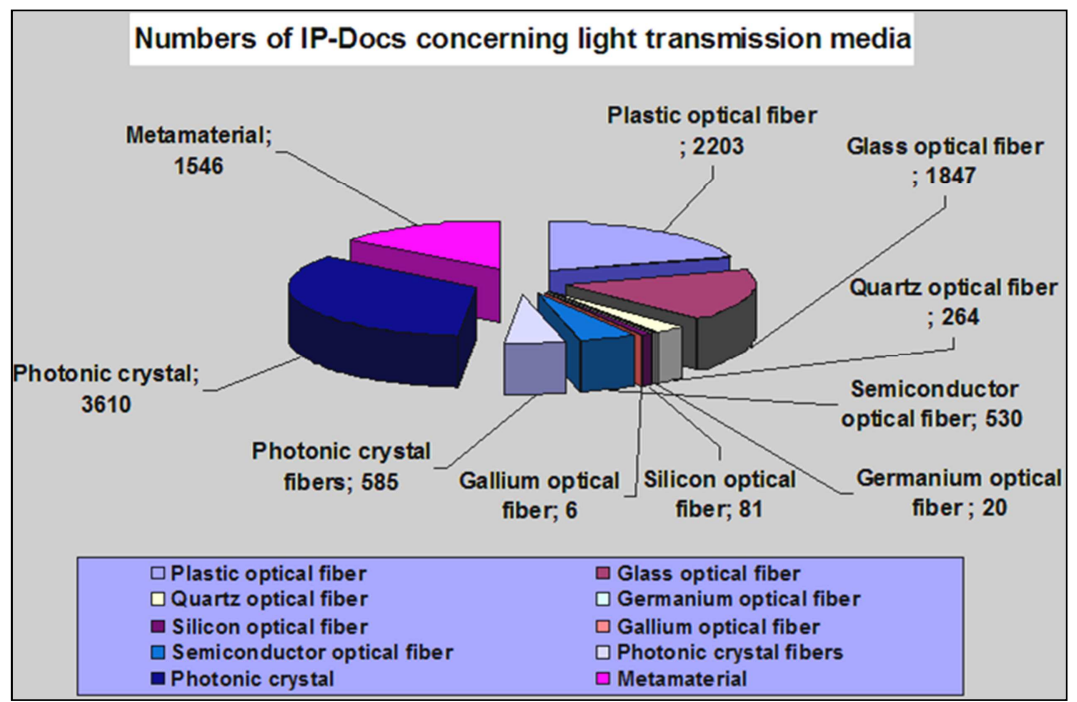

Figure 13. Numbers of IP-Docs concerning Light Transmission media. 
On-off keying has been the commonly used modulation format in optical communications. In the last years more advanced modulation formats like phase-shift keying or even orthogonal frequency-division multiplexing have been investigated to counteract effects like dispersion that degrade the quality of the transmitted signal.

Photonic systems are used for optical communication systems. This area of research focuses on the implementation of photonic systems like high speed photonic networks. This also includes research on optical regenerators, which improve optical signal quality. Photonic integrated circuits (PICs) are optically active integrated semiconductor photonic devices which consist of at least two different functional blocks, (gain region and a grating based mirror in a LASER).

New optical technology has accelerated the translation of remarkable new capabilities into medical practice. Presentday technology, driven by advances in LASER sources, nanophotonics, and detectors, generate enough data in 1 hour to fill the contents of 10, 24-volume encyclopedias.

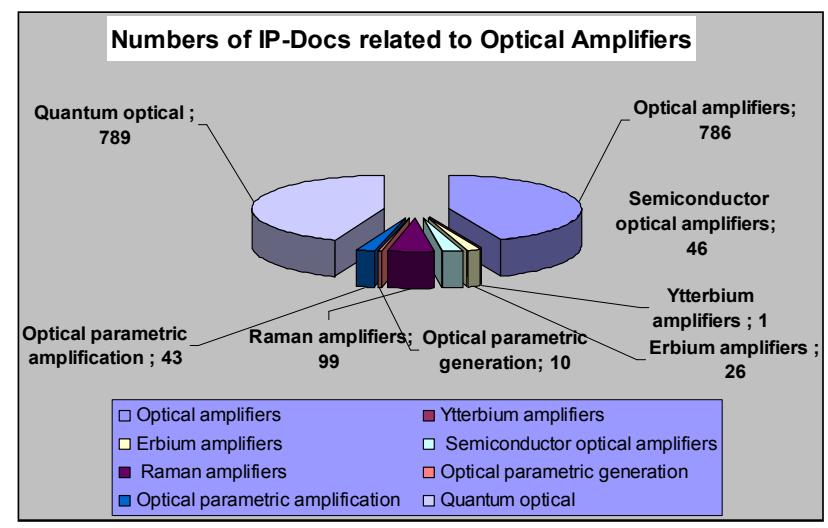

Figure 14. Numbers of IP-Docs related to Optical Amplifiers.
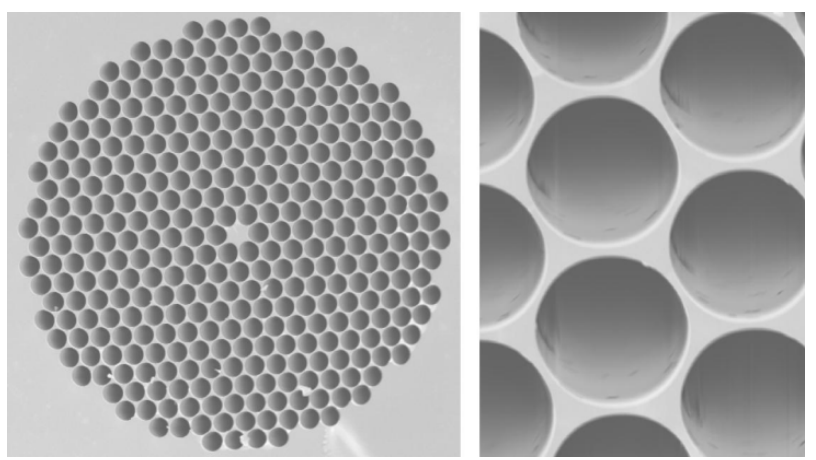

Figure 15. SEM micrographs of US Naval Research Laboratory-produced photonic-crystal fiber. Left: The diameter of the solid core at the center of the fiber is $5 \mu \mathrm{m}$. Right: The diameter of the holes is $4 \mu \mathrm{m}$. US Navy, NRL [14].

These devices are responsible for commercial successes of optical communications and the ability to increase the available bandwidth without significant cost increases to the end user, through improved performance and cost reduction that they provide. The most widely deployed PICs are based on Indium Phosphide material system. Silicon Photonics is an active area of research.

\section{How Patent-Mappings Related to Lasers and Photonics Help us to Plan the Future}

Patent-mappings are important tools, to predict the future technological, industrial, economic and even social trends in the emerging global markets and in our case-study, the potential trends in Photonic Communications Systems and Networks Markets. If we look carefully the content and the messages within the prepared diagrams, we can recognize the foci of interest of the above mentioned technologies and of their components. The number of patent applications, their continuity in time, the employment of innovative variants of a kernel Technology etc. is a very important set of factors, predicting more or less its orbit. This information is visualized on appropriate graphs, supporting positively our decision making.

Let's take an important example, the Optical MIMO Communication Systems. An optical multiple-input multipleoutput (MIMO) communication system uses multiple LEDbased transmitters and multiple receivers to transfer parallel streams of data. As compared to a single-transmitter singlereceiver system using the same amount of signal power, a MIMO system can provide higher data rates with fewer transmission errors and better reliability. There are three different types of optical MIMO systems:

The $\lambda$-MIMO is implemented using a single luminary composed of LEDs that emit different colors of light. Each LED acts as a different transmitter. Thus the parallel data streams can be transmitted over different colors of light. The receiver for this system implements optical filter to recover the signals transmitted over each color.

The $s-M I M O$ is implemented using multiple luminaries that are placed at different locations on the ceiling. Each luminary is composed of the same type of LED and thus emits the same color of light. In this case, the data streams are separated spatially because they each originate at a different spatial location. A 'camera-like' receiver can then separate the different signal streams and recover data.

The h-MIMO: This system is a hybrid of the above two systems. It uses multiple luminaries, each composed of different colored LEDs to transmit signals that are separated in color and space. A 'camera-like' receiver that can distinguish different colors can then separate the different signal streams and recover data.

Recently, Hamamatsu Photonics K. K. Laser Group has developed the Fiber Disk Laser. This unique design is so named because the optical fiber is coiled into the shape of a flat disk. This special geometry brings several performance benefits (Figure 16). The result is a source that is capable of delivering high-power output while retaining the attractive qualities of lower-power, conventional fiber LASERs. In contrast to conventional designs in which the pump light is delivered through the ends or sides of a longitudinal fiber, the 
coiled-disk-pumping scheme offers a much larger area for introducing pump light. As a consequence, the design is compatible with wide-area, high-power laser diode bars, which consist of a linear array of single emitters.

This compatibility means that the pump power can be significantly increased at low cost, which opens the door to cost-effective and convenient scaling of fiber laser output power. Furthermore, flat heat sinks can be attached to the top and bottom surfaces of the fiber disk for convenient and effective cooling.

This example explains why by following the "innovation path", paved by Patent-applications and granted Patents, we are able to create continuously new and more innovative solutions, leading to a perpetual motion of the human intelligence and inventivity and, thus, to economic growth and social progress [16]-[26].

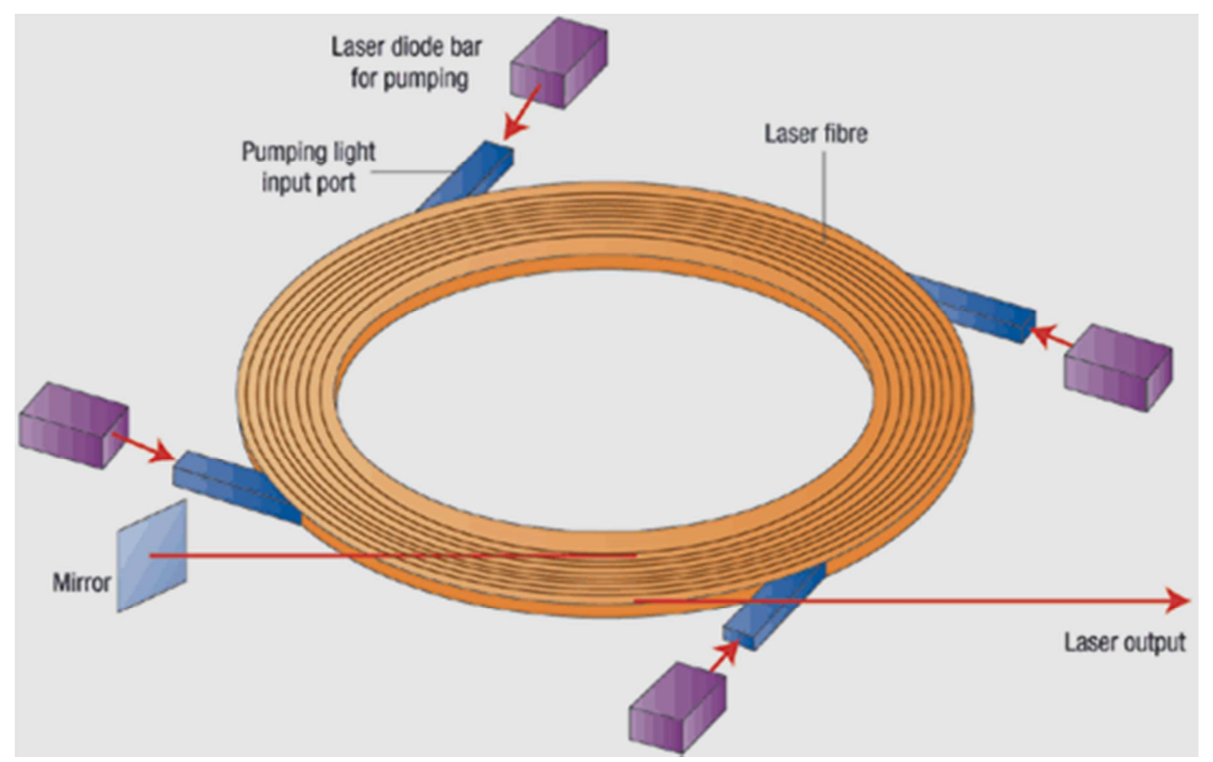

Figure 16. Schematic of the Fiber Disk Laser [15], [16].

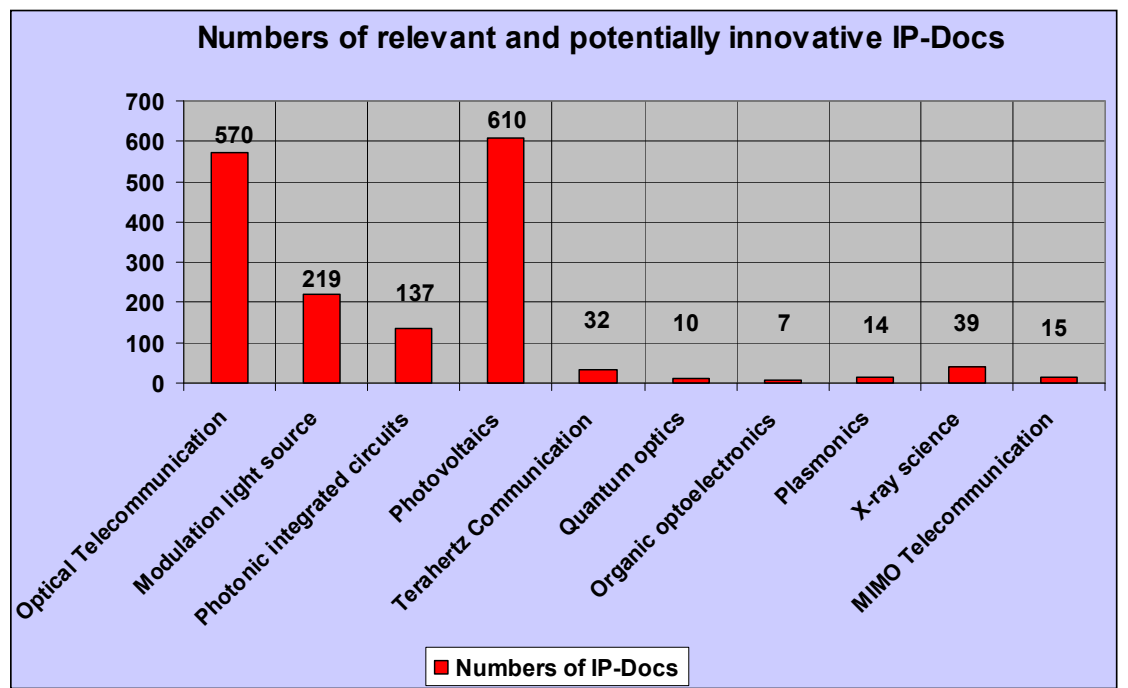

Figure 17. Numbers of relevant and potentially innovative IP-Docs related to some very important and innovative aspects of modern Optical Communications.

\section{Some Emerging Medical Applications of Photonics}

Continuing advances in several critical areas of technology have dramatically increased the capabilities of biomedical optical instrumentation and herald a new era of innovation in biomedical optics, leading to improvements in treating many types of diseases. New optical sources and materials, imaging devices, microfluidic technologies, and detection methods will provide remarkable increases in speed, sensitivity, and precision for biomedical optical instrumentation. With the advent of rapid and much-lower-cost methods for whole genome sequencing, many of which rely on optical methods, there is a possibility that a wide range of correlations between genetic makeup and disease predilection can be detected earlier, and appropriate interventions put in place.

The new generation sequencers combine nanotechnology with photonics to achieve this remarkable increase in speed. These instruments will require further investment in research 
to develop higher-speed and sensitivity CCD cameras, more efficient labeling dyes, high-speed software to extract quantitative information from large, high-resolution images, and nanophotonic structures optimized to localize the fluorescent signals from the individual DNA strands.
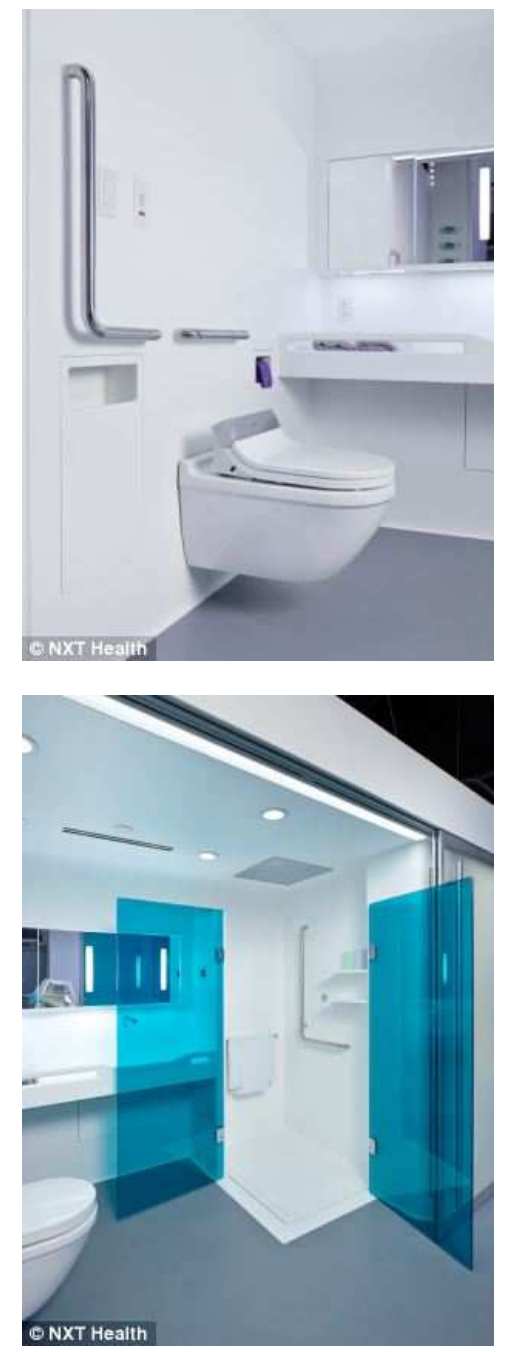

Figure 18. The bathroom is an adaptable design that features a sliding door system which can be reconfigured based on care needs [17], [18].

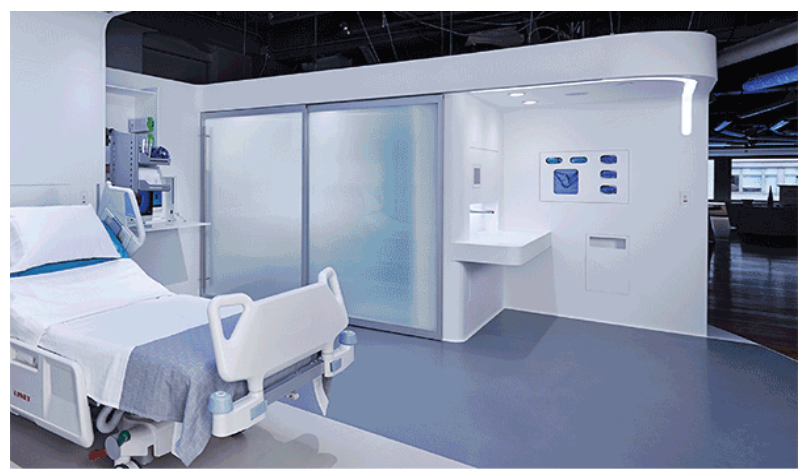

Figure 19. A typical "open" Hospital Smart-room [16].

Microfluidics and Robotics combined with optics provide the technology to create arrays of tissue samples on slides that can be automatically laser scanned and analyzed after exposure to fluorescently labeled antibodies which attach to tumor-specific proteins.

These tissue arrays are analyzed using laser scanners and automated image analysis of the digital images. Drug interactions and molecular structures can be studied across hundreds of diseased and healthy patient samples all located on a single slide. This technology has the potential for greatly accelerating the drug development process and reducing development costs.

The development of new drugs based on small molecules is often limited by the rate at which candidate molecules can be screened for their therapeutic effect on target cell cultures. In recent years, optical technology has been combined with robotics to provide the ability to screen hundreds of thousands of drug candidates per day, dramatically accelerating the drug discovery process. This highthroughput screening technology relies on robotic samplehandling automation for the precise and rapid parallel processing of multiple samples as well as on optical technology for high-speed quantitative data collection.

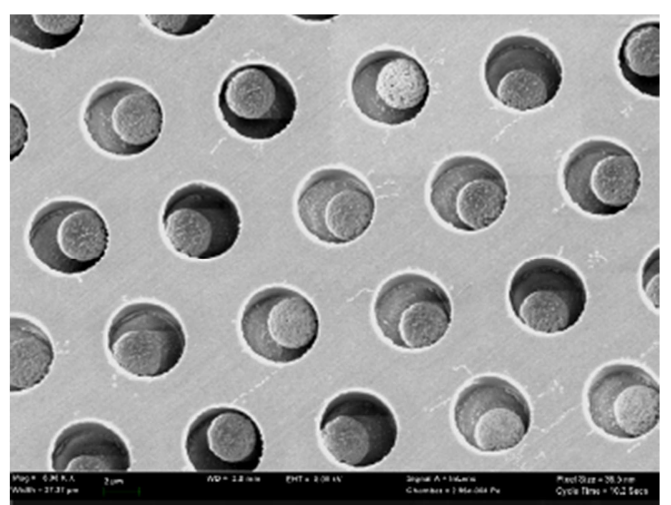

Figure 20. Reaction wells approximately 5 microns in diameter contain a single bead coated with antibodies that trap a single target protein molecule [27].

Optical methods, including fluorescence, bioluminescence, and colorimetry, are used to identify and count viable and nonviable cells affected by the candidate compounds, determine activated molecular pathways in target cells, and detect the overall cellular response to potential smallmolecule drugs. An example of an approach that allows very low concentrations of proteins to be detected by actually counting the protein molecules individually is presented in Figure 20.

A new generation of flow Cytometry instruments combines' optical detection with mass spectrometry. This new technology promises to allow a status check of a patient's immune system by simultaneously quantifying all of the major cellular constituents. When combined with other advanced proteomic technologies, including tissue microarrays and protein mass spectrometry, the CyTOF instrument outputs are presented in Figure 21. Dual Energy CT and Quantitative Image Analysis, Biomedical Optics in Regenerative Medicine and in basic Research etc. are completing the present emerging "armory" of diagnostic and therapeutic methods. 


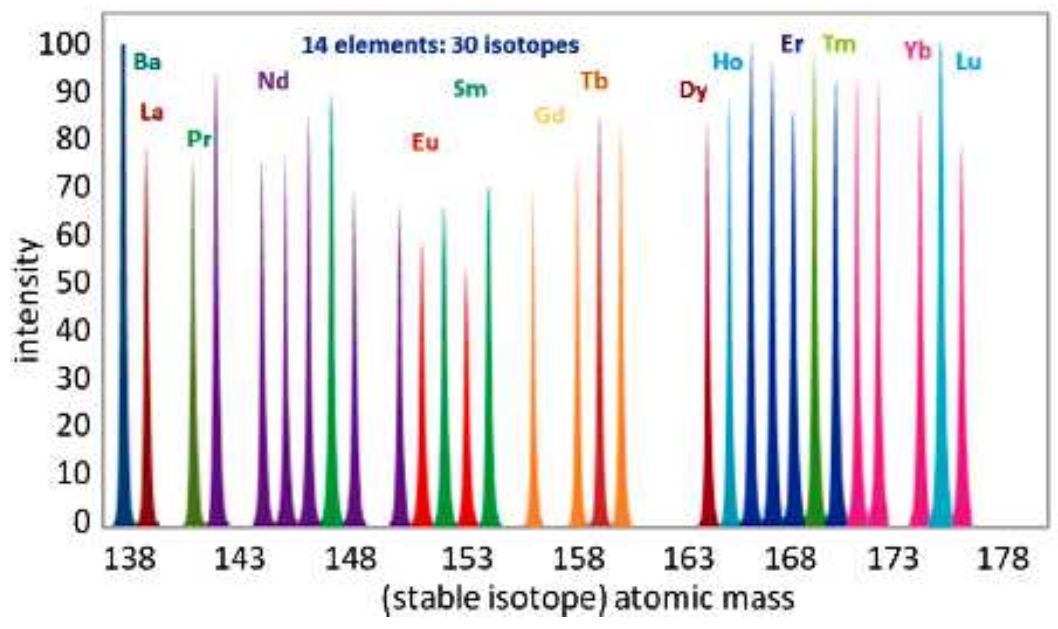

Figure 21. A CYTOF instrument, which extends the capability of multi-parameter flow Cytometry by Atomic Mass Spectrometry to measure up to 100 biomarkers simultaneously in single cells at a rate of 1,000 cells per second [28].

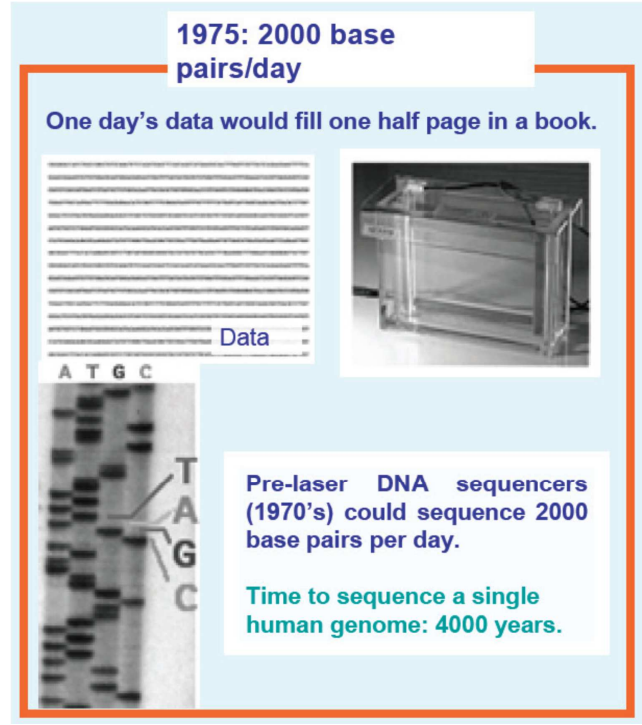

\section{Today: two billion base pairs/day}

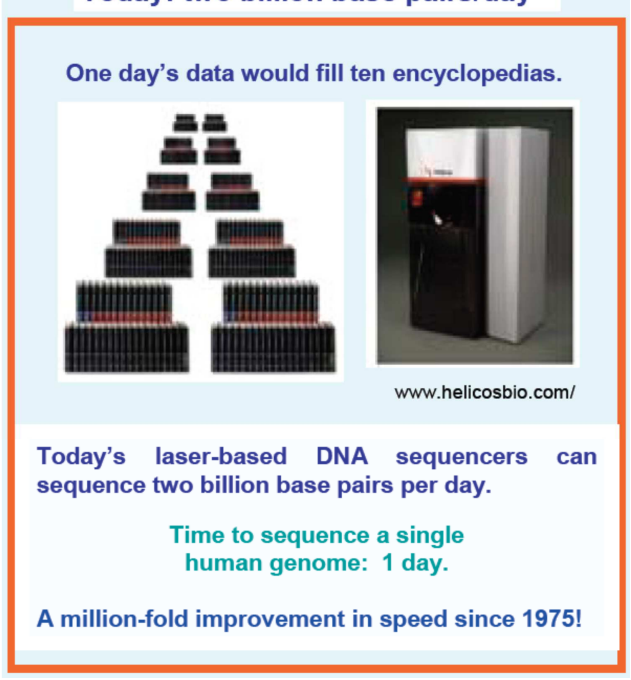

Figure 22. The optical Technologies have increased the speed of sequencing by a factor of $10^{6}$ between 1975 and today [29], [30].

\section{Concluding Remarks}

Cost, lack of Training and short-term focusing, lead to culde-sac. The Hospital-Board must realize that the world is changing dramatically; in order to survive, they have to offer smart, innovative, efficient and above all affordable and costeffective Health-care.

Overload and Cognitive Stacking negatively affects the health and employment satisfaction of Healthcare workers. It is a work environment wherein Nurses are over-whelmed with Information, Data and Distractions that ultimately can result in job dissatisfaction, burn-out and injury to a Patient. And this overload is relentlessly present, every minute, every hour, every shift, every week, for a life span...

However, the existing and emerging Biomedical Technology is a major ally of the Health-care personnel. Currently, Biomedical Technology extends and enhances the senses of the physician and thus increases the ability of the physician to diagnose disease. It is understandable that optics and imaging have played a critical role in improving health care, over the past 100 years, allowing for the clinician to see bacteria and microbial parasites led to the development of antibiotics. Direct imaging of skeleton and organs with x-ray aided in observing and setting bone fractures and diagnosing traumatic injuries to other organs. LASER based flow cytometers provide detailed quantification of critical blood cell types and state-of-the-art optical technologies increase the sensitivity and specificity of measurements, far beyond the physician's senses.

Age-related macular degeneration (AMD) and diabetic retinopathy (DR) are two of the leading causes of blindness, particularly in older patients. Laser-based surgical and drug therapies can slow the disease progression, particularly if the Disease is detected prior to major damage to the retina. Optical coherence tomography (OCT), can probe beneath the surface of the retina, providing a method for precise subsurface imaging. Image-Guided Surgery, Dual Energy CT and Quantitative Image Analysis constitute important 
contemporary photonics-based diagnostic and therapeutic methods.

Advances in Technology and the application of new instruments often provide the basis for further insights and discoveries that lead to a deeper understanding of the causes and the molecular basis of diseases. Significant improvements in optical technology have dramatically increased the ability to measure and study biological processes in both in vitro and in vivo environments.

Understanding and controlling the immune system are thus among the major challenges facing modern medicine today. Optical instrumentation will continue to be the principal enabling technology, allowing advances in the understanding of the immune system.

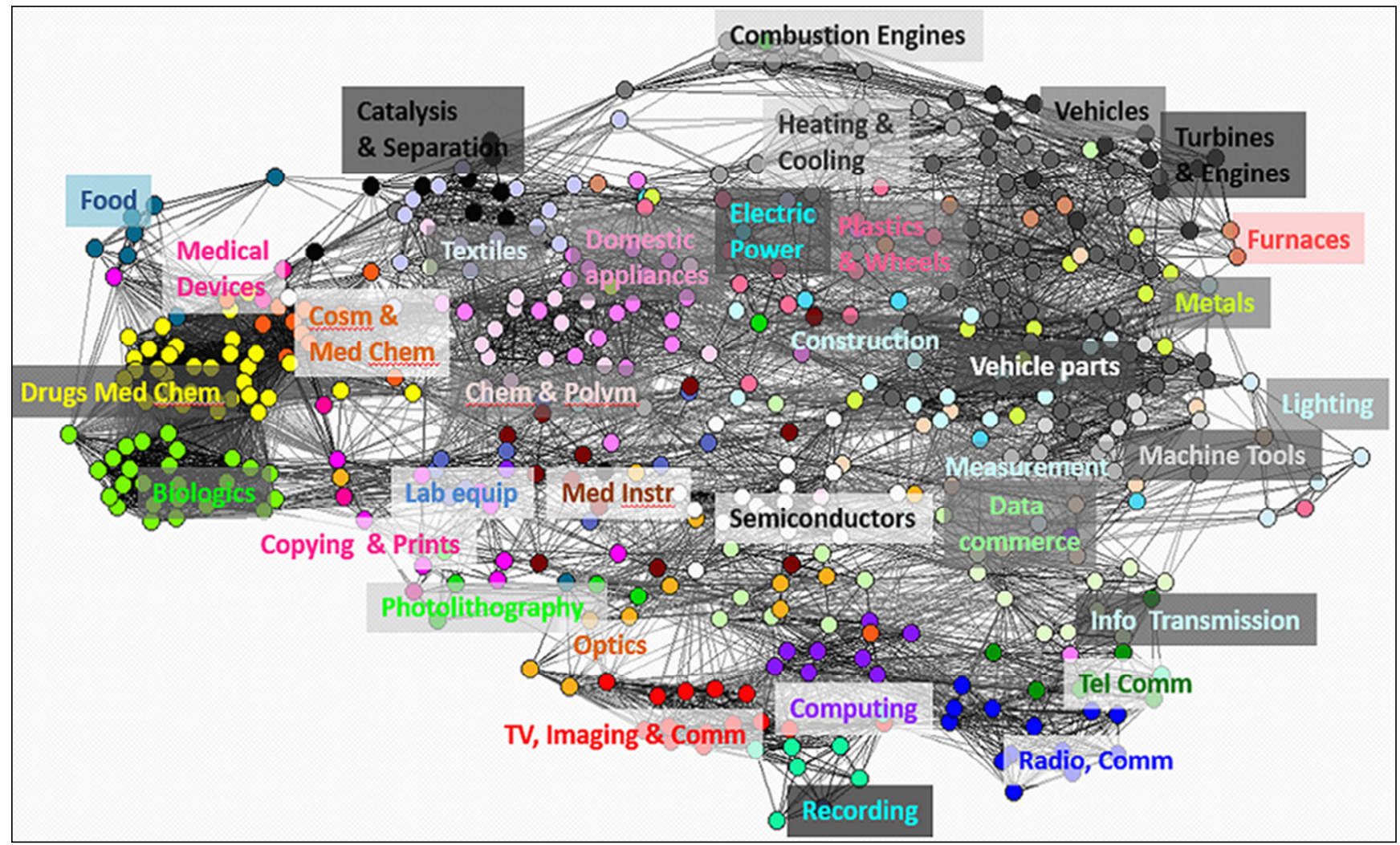

Figure 23. Global Patent Map reveals the Structure of Technological Progress presently, MIT Technology Review 2/9/13. [31].

\section{References}

[1] The Amplion Smart (er) Room of the Future: IBM and UPMC Partnership to Make 'Smart' Patient Room Even Smarter, The IBM-UPMC University of Pittsburgh Medical-Center, July $28^{\text {th }} 2010$.

[2] Katie Hollar, The 20 Most Popular EMR Software Solutions, EHR \& Medical Practice Management software advice for doctors and staff, Capterra Medical Software Blog, January $25^{\text {th }}, 2012$.

[3] Xerox survey finds patents unclear on EHR, Comparison Charts, Data and Statistics on Business, Economics, Finance, Technology, Finance, Healthcare \& More, May $19^{\text {th }} 2014$.

[4] The Princess Alexandra Hospital (PAH) became Australia's first large-scale Digital Hospital. Queensland Government, Metro South Health, November 2015.

[5] Abraham Gomez, Pourquoi le «BigData » en génomique? Schmidt, S. (2012). Les 3 V du Big Data: Volume, Vitesse et Variété. Paru dans JDN, l'économie demain le 31 mai 2012.
[6] Krisa Tailor, All-Payer Claims Databases (APCDs) set stage for data-driven healthcare \& big analytics, SAS, November $28^{\text {th }}, 2012$.

[7] Predictive Analytics in Practice: A Harvard Business Review Insight Center Report, Harvard Business Publishing, 2014.

[8] The Hospital Room of the Future: A patient-centered design could reduce infections, falls, errors - and ultimately costs, Bamboo Innovator, Nov. 17, 2013.

[9] Sara Heath, Patient Engagement Technology: Value-Based Care Key 2018 Trends, Patient Engagement Hit, December $18,2017$.

[10] Patient Engagement Framework: Building an organizational capability in patient engagement, Micromass Communications Inco.

[11] Burt J. et al., Improving patient experience in primary care: a multimethod programme of research on the measurement and improvement of patient experience, Southampton (UK): NIHR Journals Library; 2017 Apr. PMID: 28654227.

[12] Reducing the personnel work-load by introducing targeted improvements: Memorial Hermann, Houston Hospitals, Institutes \& Centers. 
[13] LSCC Adult Health and Wellness at Lake Aire Visit Workflow: A typical Clinical-workflow diagram. https://www.slideshare.net/ataveechai/clinic-workflowdiagram.

[14] The two views of a Clinical Process: https://image.slidesharecdn.com/workflowonlinepres11007135240-phpapp01/95/modeling-clinical-workflow-2728.jpg?cb=1317995745.

[15] UPMC/University of Pittsburgh Schools of the Health Sciences. http://www.upmc.com/media/NewsReleases/2010/Pages/ibmupmc-partner-make-Smart-Patient-room-even-Smarter.aspx.

[16] Smart Lighting Shows Off in Patient Room 2020 https://gr.pinterest.com/pin/514184482429856752/.

[17] http://www.dailymail.co.uk/health/article-2384889/PatientRoom-2020-Designers-unveil-hi-tech-visions-Hospitalfuture.htm (Next Health).

[18] PATIENT ROOM 2020: A collaborative project to design a next generation inpatient health experience. http://nxthealth.org/Patient-room-2020/.

[19] B. Spyropoulos, 50 years LASERS: In vitro Diagnostics, Clinical Applications and Perspectives, Clinical Laboratory, Nr. 3+4/2011.

[20] Laser history (1917-1996) from Einstein to present day, from microwave lasers to optical and X-ray LASERS, discovered in space and lasers in astronomy http://laserstars.org/history.

[21] Understanding Industrial Property, WIPO, http://www.wipo.int/edocs/pubdocs/en/wipo_pub_895_2016.p df.

[22] http://www.nrl.navy.mil/techtransfer/fs.php?fs_id=97.
[23] Optical MIMO Communication Systems https://www.bu.edu/smartlighting/research/optical-mimo/.

[24] Fiber Disk Laser explained: Hamamatsu Photonics Laser Group:

http://www.nature.com/nphoton/journal/vsample/nsample/fig tab/nphoton.2006.6_F1.html Nature Photonics sample, pp14 15 (2006)

[25] $10^{\text {th }}$ Anniversary Nature Photonics Vol. 11, No. 1 (January 2017), http://www.nature.com/nphoton/focus/index.html.

[26] Mohammad Ali Khalighi, Murat Uysal, Survey on Free Space Optical Communication: A Communication Theory Perspective, pp. 2231-2258 26/6 2014 http://ieeexplore.ieee.org/document/6844864/.

[27] L. Kay et al. Patent Overlay Mapping: Visualizing Technological Distance, Cornell Libr. OCT 2013 https://arxiv.org/abs/1208.4380.

[28] Subbaraman, N. 2010. "Detecting Single Cancer Molecules", Tech. Review at:

http://www.technologyreview.com/biomedicine/25462/.

[29] DVS Sciences, Inc. at http://www.dvssciences.com/index.xhtml.

[30] Congressional briefing by Thomas Baer, Executive Director, http://portal.acs.org/preview/fileFetch/C/CNBP_026401/pdf/C NBP_026401.pdf Stanford Photonics Research Center, Stanford University, Palo Alto, Calif. November 30, 2010.

[31] Global Patent Map Reveals the Structure of Technological Progress www.technologyreview.com/s/518991/global-patentmap-reveals-the-structure-of-technological-progress/ MIT Technology Review 2/9/13. 